Math. Struct. in Comp. Science (2017), vol. 27, pp. 143-156. (c) Cambridge University Press 2015. This is an Open Access article, distributed under the terms of the Creative Commons Attribution licence (http://creativecommons.org/licenses/by/3.0/), which permits unrestricted re-use, distribution, and reproduction in any medium, provided the original work is properly cited.

doi:10.1017/S0960129515000134 First published online 11 May 2015

\title{
Fast circular dictionary-matching algorithm
}

TANVER ATHAR ${ }^{\dagger}$, CARL BARTON ${ }^{\dagger}$, WIDMER BLAND

JIA GAO ${ }^{\dagger}$, COSTAS S. ILIOPOULOS ${ }^{\dagger}, \mathrm{CHANG} \mathrm{LIU}^{\dagger}$ and

SOLON P. PISSIS ${ }^{\dagger}$

${ }^{\dagger}$ Department of Informatics, King's College London, London, UK

${ }^{\ddagger}$ Department of Computing and Software, McMaster University, Hamilton, Canada

Received 9 February 2014; revised 5 March 2015

Circular string matching is a problem which naturally arises in many contexts. It consists in finding all occurrences of the rotations of a pattern of length $m$ in a text of length $n$. There exist optimal worst- and average-case algorithms for circular string matching. Here, we present a suboptimal average-case algorithm for circular string matching requiring time $\mathcal{O}(n)$ and space $\mathcal{O}(m)$. The importance of our contribution is underlined by the fact that the proposed algorithm can be easily adapted to deal with circular dictionary matching. In particular, we show how the circular dictionary-matching problem can be solved in average-case time $\mathcal{O}(n+M)$ and space $\mathcal{O}(M)$, where $M$ is the total length of the dictionary patterns, assuming that the shortest pattern is sufficiently long. Moreover, the presented average-case algorithms and other worst-case approaches were also implemented.

Experimental results, using real and synthetic data, demonstrate that the implementation of the presented algorithms can accelerate the computations by more than a factor of two compared to the corresponding implementation of other approaches.

\section{Introduction}

In order to provide an overview of our results and algorithms, we begin with a few definitions generally following (Smyth 2003). We think of a string $x$ of length $n$ as an array $x[0 \ldots n-1]$, where every $x[i], 0 \leqslant i<n$, is a letter drawn from some fixed alphabet $\Sigma$ of size $\sigma=|\Sigma|$. The empty string of length 0 is denoted by $\varepsilon$. A string $x$ is a factor of a string $y$ if there exist two strings $u$ and $v$, such that $y=u x v$. Let the strings $x, y, u$, and $v$, such that $y=u x v$. If $u=\varepsilon$, then $x$ is a prefix of $y$. If $v=\varepsilon$, then $x$ is a suffix of $y$. Let $x$ be a non-empty string of length $n$ and $y$ be a string. We say that there exists an occurrence of $x$ in $y$, or, more simply, that $x$ occurs in $y$, when $x$ is a factor of $y$. Every occurrence of $x$ can be characterized by a position in $y$. Thus we say that $x$ occurs at the starting position $i$ in $y$ when $y[i . . i+n-1]=x$. We define the $i$ th prefix to be the prefix ending at position $i$ i.e. $x[0 \ldots i], 0 \leqslant i<n$. On the other hand, the $i$ th suffix is the suffix starting at position $i$ i.e. $x[i . . n-1], 0 \leqslant i<n$.

A circular string of length $m$ can be viewed as a traditional linear string which has the left- and right-most symbols wrapped around and stuck together in some way. Under this notion, the same circular string can be seen as $m$ different linear strings, which would all be considered equivalent. Given a string $x$ of length $m$, we denote by 
$x^{i}=x[i \ldots m-1] x[0 \ldots i-1], 0<i<m$, the $i$ th rotation of $x$ and $x^{0}=x$. Consider, for instance, the string $x=x^{0}=$ abababbc; this string has the following rotations: $x^{1}=$ bababbca, $x^{2}=$ ababbcab, $x^{3}=$ babbcaba, $x^{4}=$ abbcabab, $x^{5}=$ bbcababa , $x^{6}=$ bcababab, $x^{7}=$ cabababb.

Here, we consider the problem of finding occurrences of a pattern $x$ of length $m$ with circular structure in a text $t$ of length $n$ with linear structure. This is the problem of circular string matching; it has been considered in Lothaire (2005), where an $\mathcal{O}(n)$-time algorithm was presented. A naive solution with quadratic complexity consists in applying a classical algorithm for searching a finite set of strings after having built the trie of rotations of $x$. The approach presented in Lothaire (2005) consists in preprocessing $x$ by constructing a suffix automaton of the string $x x$, by noting that every rotation of $x$ is a factor of $x x$. Then, by feeding $t$ into the automaton, the lengths of the longest factors of $x x$ occurring in $t$ can be found by the links followed in the automaton in time $\mathcal{O}(n)$. In Fredriksson and Grabowski (2009), the authors presented an optimal average-case algorithm for circular string matching, by also showing that the average-case lower bound for single string matching of $O\left(n \log _{\sigma} m / m\right)$ also holds for circular string matching. Very recently, in Chen et al. (2013), the authors presented two fast average-case algorithms based on wordlevel parallelism. The first algorithm requires average-case time $\mathcal{O}\left(n \log _{\sigma} m / w\right)$, where $w$ is the number of bits in the computer word. The second one is based on a mixture of word-level parallelism and $q$-grams. The authors showed that with the addition of $q$-grams, and by setting $q=\mathcal{O}\left(\log _{\sigma} m\right)$, an optimal average-case time of $\mathcal{O}\left(n \log _{\sigma} m / m\right)$ is achieved.

Given a set $\mathcal{D}$ of $d$ pattern strings, the dictionary-matching problem is to index $\mathcal{D}$ such that for any online query text $t$, one can quickly find the occurrences of any pattern of $\mathcal{D}$ in $t$. This problem has been well studied in the literature (Aho and Corasick 1975; Chan et al. 2007), and an index taking optimal space and simultaneously supporting timeoptimal queries is achieved (Belazzougui 2010; Hon et al. 2010). In some applications in computational molecular biology, such as, for instance, pattern matching of a collection of viral sequences, we are interested in searching for, not only the original patterns in $\mathcal{D}$, but also all of their rotations. This is the problem of circular dictionary matching.

A variant of this problem for an offline query text was first discussed in Iliopoulos and Rahman (2008). The authors proposed two index data structures, namely CPI-I and CPI-II. CPI-I can be constructed in time and space $\mathcal{O}\left(n \log ^{1+\varepsilon} n\right)$, and an online pattern query can be answered in time $\mathcal{O}(m \log \log n+O c c)$, where $O c c$ is the number of occurrences. However, CPI-I involves constructing two suffix trees (Weiner 1973) as well as a complex range-search data structure. Hence, it is suspected that, despite a good theoretical time bound, the practical performance of CPI-I would not be very good both in terms of time and space. CPI-II on the other hand uses a suffix array (Manber and Myers 1993), which is much more space-efficient than a suffix tree and does not require the range-search data structure. CPI-II is conceptually much simpler and can be built in time $\mathcal{O}(n)$ and requires $\mathcal{O}(n \log n)$ bits of space; an online pattern query can then be answered in time $\mathcal{O}(m \log n+O c c)$.

The problem for any online query text was studied in Hon et al. (2011). The authors proposed a variant of suffix tree, called circular suffix tree and showed that it can be 
compressed into succinct space. With a tree structure augmented to a circular pattern matching index called circular suffix array, the circular suffix tree can be used to solve the circular dictionary-matching problem efficiently. Very recently, in Hon et al. (2013) the authors proposed the first algorithm for the efficient construction of the circular suffix tree, which requires time $\mathcal{O}(M \log M)$ and $O(M \log \sigma+d \log M)$ bits of working space, where $M$ is the total length of the dictionary patterns.

In this article, we revisit the following two problems in the average-case setting.

CirCUlARSTRINGMATCHING

Input: a pattern $x$ of length $m$ and a text $t$ of length $n>m$

Output: all factors $u$ of $t$ such that $u=x^{i}, 0 \leqslant i<m$

Circular DictionaryMatching

Input: a set $\mathcal{D}=\left\{x_{0}, x_{1} \ldots, x_{d-1}\right\}$ of patterns of total length $M$ and a text $t$ of length $n$, such that $n>\left|x_{j}\right|, 0 \leqslant j<d$

Output: all factors $u$ of $t$ such that $u=x_{j}^{i}, 0 \leqslant j<d, 0 \leqslant i<\left|x_{j}\right|$

\subsection{Our contribution}

We present a new suboptimal average-case algorithm for circular string matching requiring time $\mathcal{O}(n)$ and space $\mathcal{O}(m)$. We show how it can be extended to solve the circular dictionarymatching problem in average-case time $\mathcal{O}(n+M)$ and space $\mathcal{O}(M)$, assuming that the shortest pattern is sufficiently long. Furthermore, we implement the presented average-case algorithms and the index data structure CPI-II presented in Iliopoulos and Rahman (2008) for an offline query text. Experimental results, using real and synthetic data, demonstrate that the implementation of the algorithms proposed here can accelerate the computations by more than a factor of two compared to the corresponding implementation of CPI-II. A preliminary version of this work appeared in Barton et al. (2013).

\section{Properties of the partitioning technique}

In this section, we give a brief outline of the partitioning technique in general; and then show some properties of the version of the technique we use for our algorithms. The partitioning technique, introduced in Wu and Manber (1992), and in some sense earlier in Rivest (1976), is an algorithm based on filtering out candidate positions that could never give a solution to speed-up string-matching algorithms. An important point to note about this technique is that it reduces the search space but does not, by design, verify potential occurrences. To create a string-matching algorithm filtering must be combined with some verification technique. The idea behind the partitioning technique was initially proposed for approximate string matching, but here we show that this can also be used for exact circular string matching.

The idea behind the partitioning technique is to partition the given pattern in such a way that at least one of the fragments must occur exactly in any valid approximate occurrence of the pattern. It is then possible to search for these fragments exactly to give 

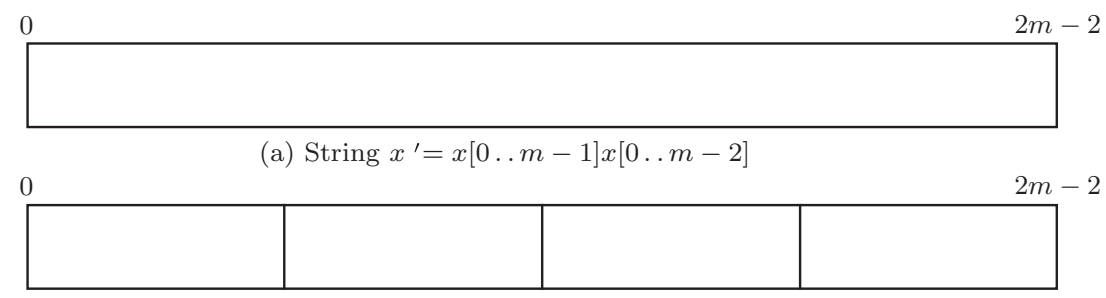

(b) Partition $x^{\prime}$ in 4 fragments of length $\ell_{f}=(2 m-1) / 4$
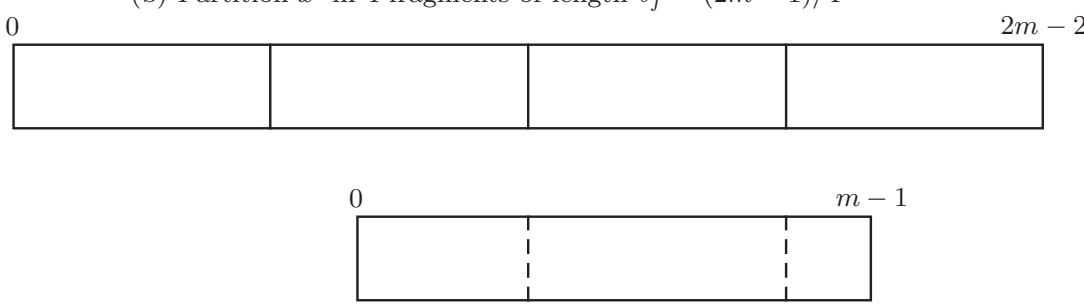

(c) $m>2 \ell_{f}$; any factor of length $m$ of $x^{\prime}$ contains at least one of the 4 fragments

Fig. 1. Illustration of Lemma 2.1. (a) String $x^{\prime}=x[0 \ldots m-1] x[0 \ldots m-2]$. (b) Partition $x^{\prime}$ in four fragments of length $\ell_{f}=(2 m-1) / 4$. (c) $m>2 \ell_{f}$; any factor of length $m$ of $x^{\prime}$ contains at least one of the four fragments.

a set of candidate occurrences of the pattern. It is then left to the verification portion of the algorithm to check if these are valid approximate occurrences of the pattern. It has been experimentally shown that this approach yields very good practical performance on large-scale datasets (Frousios et al. 2010), even if it is not theoretically optimal.

For exact circular string matching, for an efficient solution, we cannot simply apply well-known exact string-matching algorithms, as we must also take into account the rotations of the pattern. We can, however, make use of the partitioning technique and, by choosing an appropriate number of fragments, ensure that at least one fragment must occur in any valid exact occurrence of a rotation. Lemma 2.1 together with the following fact provide this number.

Fact 1. Let $x$ be a string of length $m$. Any rotation of $x$ is a factor of $x^{\prime}=x[0 \ldots m-$ $1] x[0 . . m-2]$; and any factor of length $m$ of $x^{\prime}$ is a rotation of $x$.

Lemma 2.1. Let $x$ be a string of length $m$. If we partition $x^{\prime}=x[0 \ldots m-1] x[0 \ldots m-2]$ in four fragments of length $\lfloor(2 m-1) / 4\rfloor$ and $\lceil(2 m-1) / 4\rceil$, at least one of the four fragments is a factor of any factor of length $m$ of $x^{\prime}$.

Proof. Let $\ell_{f}$ denote the length of the fragment. If we partition $x^{\prime}$ in at least four fragments of length $\lfloor(2 m-1) / 4\rfloor$ and $\lceil(2 m-1) / 4\rceil$, we have that

$$
\ell_{f} \leqslant(2 m-1) / 4
$$

which gives $2 m>4 \ell_{f}$ and $m>2 \ell_{f}$. Therefore, any factor of length $m$ of $x^{\prime}$, and, by Fact 1 , any rotation of $x$, must contain at least one of the fragments. For a graphical illustration of this proof inspect Figure 1. 


\section{Circular string matching via filtering}

In this section, we present CSMF, a new suboptimal average-case algorithm for exact circular string matching via filtering. It is based on the partitioning technique and a series of practical and well-established data structures.

\subsection{Longest common extension}

First, we describe how to compute the longest common extension, denoted by Ice, of two suffixes of a string in constant time. Ice queries are an important part of the algorithms presented later on.

Let SA denote the array of positions of the sorted suffixes of string $x$ of length $n$, i.e. for all $1 \leqslant r<n$, we have $x[\mathrm{SA}[r-1] \ldots n-1]<x[\mathrm{SA}[r] \ldots n-1]$. The inverse iSA of the array $\mathrm{SA}$ is defined by iSA[SA $[r]]=r$, for all $0 \leqslant r<n$. Let $\operatorname{Icp}(r, s)$ denote the length of the longest common prefix of the strings $x[\mathrm{SA}[r] \ldots n-1]$ and $x[\mathrm{SA}[s] \ldots n-1]$, for all $0 \leqslant r, s<n$, and 0 otherwise. Let LCP denote the array defined by $\operatorname{LCP}[r]=\operatorname{Icp}(r-1, r)$, for all $1<r<n$, and $\operatorname{LCP}[0]=0$. We perform the following linear-time and linear-space preprocessing:

- Compute arrays SA and iSA of $x$ (Nong et al. 2009).

- Compute array LCP of $x$ (Fischer 2011).

- Preprocess array LCP for range minimum queries, we denote this by RMQ and Heun 2011).

With the preprocessing complete, the Ice of two suffixes of $x$ starting at positions $p$ and $q$ can be computed in constant time in the following way (Ilie et al. 2010):

$$
\operatorname{LCE}(x, p, q)=\operatorname{LCP}\left[\operatorname{RMQ}_{\mathrm{LCP}}(\mathrm{iSA}[p]+1, \mathrm{iSA}[q])\right] .
$$

Example 3.1. Let the string $x=$ abbababba. The following table illustrates the arrays SA, iSA, and LCP for $x$.

\begin{tabular}{rlllllllll}
\hline$i$ & 0 & 1 & 2 & 3 & 4 & 5 & 6 & 7 & 8 \\
\hline$x[i]$ & $\mathrm{a}$ & $\mathrm{b}$ & $\mathrm{b}$ & $\mathrm{a}$ & $\mathrm{b}$ & $\mathrm{a}$ & $\mathrm{b}$ & $\mathrm{b}$ & $\mathrm{a}$ \\
$\mathrm{SA}[i]$ & 8 & 3 & 5 & 0 & 7 & 2 & 4 & 6 & 1 \\
$\mathrm{iSA}[i]$ & 3 & 8 & 5 & 1 & 6 & 2 & 7 & 4 & 0 \\
$\mathrm{LCP}[i]$ & 0 & 1 & 2 & 4 & 0 & 2 & 3 & 1 & 3 \\
\hline
\end{tabular}

We have $\operatorname{LCE}(x, 1,2)=\operatorname{LCP}\left[\operatorname{RMQ}_{\mathrm{LCP}}(\mathrm{iSA}[2]+1\right.$, iSA $\left.[1])\right]=\operatorname{LCP}\left[\operatorname{RMQ}_{\mathrm{LCP}}(6,8)\right]=1$, implying that the Ice of bbababba and bababba is 1 .

\subsection{Algorithm CSMF}

Given a pattern $x$ of length $m$ and a text $t$ of length $n>m$, an outline of algorithm CSMF for solving the CircularStringMatching problem is as follows. 
1. Construct the string $x^{\prime}=x[0 \ldots m-1] x[0 \ldots m-2]$ of length $2 m-1$. By Fact 1 , any rotation of $x$ is a factor of $x^{\prime}$.

2. The pattern $x^{\prime}$ is partitioned in four fragments of length $\lfloor(2 m-1) / 4\rfloor$ and $\lceil(2 m-1) / 4\rceil$. By Lemma 2.1, at least one of the four fragments is a factor of any rotation of $x$.

3. Match the four fragments against the text $t$ using an Aho-Corasick automaton (Dori and Landau 2006). Let $\mathcal{L}$ be a list of size $O c c$ of tuples, where $<p_{x^{\prime}}, \ell, p_{t}>\in \mathcal{L}$ is a three-tuple such that $0 \leqslant p_{x^{\prime}}<2 m-1$ is the position where the fragment occurs in $x^{\prime}$, $\ell$ is the length of the corresponding fragment, and $0 \leqslant p_{t}<n$ is the position where the fragment occurs in $t$.

4. Compute SA, iSA, LCP, and RMQ $Q_{\mathrm{LCP}}$ of $T=x^{\prime} t$. Compute SA, iSA, LCP, and RMQ of $T_{r}=\operatorname{rev}\left(t x^{\prime}\right)$, that is the reverse string of $t x^{\prime}$.

5. For each tuple $<p_{x^{\prime}}, \ell, p_{t}>\in \mathcal{L}$, we try to extend to the right via computing

$$
\mathcal{E}_{r} \leftarrow \operatorname{LCE}\left(t, p_{x^{\prime}}+\ell, 2 m-1+p_{t}+\ell\right) ;
$$

in other words, we compute the length $\mathcal{E}_{r}$ of the longest common prefix of $x^{\prime}\left[p_{x^{\prime}}+\right.$ $\ell . .2 m-1]$ and $t\left[p_{t}+\ell \ldots n-1\right]$, both being suffixes of $T$. Similarly, we try to extend to the left via computing $\mathcal{E}_{l}$ using Ice queries on the suffixes of $T_{r}$.

6. For each $\mathcal{E}_{l}, \mathcal{E}_{r}$ computed for tuple $<p_{x^{\prime}}, \ell, p_{t}>\in \mathcal{L}$, we report all the valid starting positions in $t$ by first checking if the total length $\mathcal{E}_{l}+\ell+\mathcal{E}_{r} \geqslant m$; that is the length of the full extension of the fragment is greater than or equal to $m$, matching at least one rotation of $x$. If that is the case, then we report positions

$$
\max \left\{p_{t}-\mathcal{E}_{\ell}, p_{t}+\ell-m\right\}, \ldots, \min \left\{p_{t}+\ell-m+\mathcal{E}_{r}, p_{t}\right\} .
$$

Example 3.2. Let the pattern $x=$ GGGTCTA of length $m=7$, and the text $t=$ GataCGATACCTAGgGTGATAGAATAG. Then $x^{\prime}=$ GGGTCTAGGGTCT (Step 1). $x^{\prime}$ is partitioned in GGGT, CTA, GGG, and TCT (Step 2). Consider $<4,3,10>\in \mathcal{L}$, that is, fragment $x^{\prime}[4 \ldots 6]=$ CTA, of length $\ell=3$, occurs at starting position $p_{t}=10$ in $t$ (Step 3). Then $T=$ GGGTCTAGGGTCTGATACGATACCTAGGGTGATAGAATAG and $T_{r}=$ TCTGGGATCTGGGGATAAGATAGTGGGATCCATAGCATAG (Step 4). Extending to the left gives $\mathcal{E}_{l}=0$, since $T_{r}[9] \neq T_{r}[30]$; and extending to the right gives $\mathcal{E}_{r}=4$, since $T[7 . .10]=T[26 . .29]$ and $T[11] \neq T[30]$ (Step 5). We check that $\mathcal{E}_{l}+\ell+\mathcal{E}_{r}=7=m$, and therefore we report position 10 (Step 6):

$$
p_{t}-\mathcal{E}_{\ell}=10-0=10, \ldots, p_{t}+\ell-m+\mathcal{E}_{r}=10+3-7+4=10 ;
$$

that is, $x^{4}=$ CTAGGGT occurs at starting position 10 in $t$.

Theorem 3.1. Given a pattern $x$ of length $m$ drawn from alphabet $\Sigma, \sigma=|\Sigma|$, and a text $t$ of length $n>m$ drawn from $\Sigma$, algorithm CSMF requires average-case time $\mathcal{O}(n)$ to solve the CircularStringMatching problem.

Proof. Constructing and partitioning the string $x^{\prime}$ from $x$ can trivially be done in time $\mathcal{O}(m)$ (Steps 1-2). Building the Aho-Corasick automaton of the four fragments requires time $\mathcal{O}(m)$; and the search time is $\mathcal{O}(n+O c c)$ (Step 3) (Dori and Landau 2006). The preprocessing step for the Ice queries on the suffixes of $T$ and $T_{r}$ can be done in 
time $\mathcal{O}(n)$ (Step 4). Computing $\mathcal{E}_{l}$ and $\mathcal{E}_{r}$ for each occurrence of a fragment requires time $\mathcal{O}(\mathrm{Occ})$ (Step 5). For each extended occurrence of a fragment, we report $\mathcal{O}(\mathrm{m})$ valid starting positions, thus $\mathcal{O}(m O c c)$ in total (Step 6). Since, the expected number Occ of occurrences of the four fragments in $t$ is $4 n / \sigma^{(2 m-1) / 4}=\mathcal{O}\left(\frac{n}{\sigma^{\frac{2 m-1}{4}}}\right)$, algorithm CSMF requires average-case time $\mathcal{O}\left(\left(1+\frac{m}{\sigma^{\frac{2 m-1}{4}}}\right) n\right)$. It achieves average-case time $\mathcal{O}(n)$ iff

$$
f=\frac{4 m}{\sigma^{\frac{2 m-1}{4}}} n \leqslant c n
$$

for some fixed constant $c$. For $\sigma=2$, the maximum value of $f$ is attained at

$$
m=2 / \ln 2 \approx 2.8853
$$

and so for $\sigma>1$ we get

$$
\frac{4 m}{\sigma^{\frac{2 m-1}{4}}} n \leqslant 5.05 n
$$

\subsection{Algorithm CSMF-Simple}

In this section, we present algorithm CSMF-Simple, a more space-efficient version of algorithm CSMF. Algorithm CSMF-Simple is very similar to algorithm CSMF. The only differences are:

- Algorithm CSMF-Simple does not perform Step 4 of algorithm CSMF;

- For each tuple $\left\langle p_{x^{\prime}}, \ell, p_{t}\right\rangle \in \mathcal{L}$, Step 5 of algorithm CSMF is performed without the use of the pre-computed indexes. In other words, we compute $\mathcal{E}_{r}$ and $\mathcal{E}_{\ell}$ by simply performing letter comparisons and counting the number of mismatches occurred. The extension stops right before the first mismatch.

Fact 2. The expected number of letter comparisons required for each extension in algorithm CSMF-Simple is less than three.

Proof. Recall that on an alphabet of size $\sigma$, the probability that two random strings of length $\ell$ are equal is $(1 / \sigma)^{\ell}$. Thus, given two long strings, and setting $r=1 / \sigma$, such that $r<1$, there is probability $r$ that the initial letters are equal, $r^{2}$ that the prefixes of length two are equal, and so on. Thus, the expected number of positions to be matched before inequality occurs can be described by the summation of infinite terms

$$
S=r+2 r^{2}+\cdots=\sum_{k=1}^{\infty} k r^{k}
$$

which is bounded by $r /(1-r)^{2}<2$ for $r<1$.

Thus $S$, the expected number of matching positions, is less than two, and hence, the expected number of letter comparisons required for each extension in algorithm CSMF-Simple is less than three. 
Theorem 3.2. Given a pattern $x$ of length $m$ drawn from alphabet $\Sigma, \sigma=|\Sigma|$, and a text $t$ of length $n>m$ drawn from $\Sigma$, algorithm CSMF-Simple requires average-case time $\mathcal{O}(n)$ and space $\mathcal{O}(m)$ to solve the CircularStringMatching problem.

Proof. By Fact 2, computing $\mathcal{E}_{\ell}$ and $\mathcal{E}_{r}$ for each occurrence of a fragment requires expected time $\mathcal{O}(\mathrm{Occ})$. Therefore, algorithm CSMF-Simple requires average-case time $\mathcal{O}(n)$. The required space is reduced to $\mathcal{O}(m)$ since Step 4 of algorithm CSMF is not performed.

\section{Circular dictionary matching via filtering}

In this section, we give a generalization of our algorithm for circular string matching and show that it can easily be modified to solve the problem of circular dictionary matching. We denote this new algorithm by CDMF. Algorithm CDMF follows the same approach as before but with a few key differences. In circular dictionary matching we are given a set $\mathcal{D}=\left\{x_{0}, x_{1}, \ldots, x_{d-1}\right\}$ of patterns of total length $M$ and we must find all occurrences of the patterns in $\mathcal{D}$ or any of their rotations. To modify algorithm CSMF to solve this problem we perform Steps 1 and 2 for every pattern in $\mathcal{D}$, constructing the strings $x_{0}^{\prime}, x_{1}^{\prime}, \ldots, x_{d-1}^{\prime}$ and breaking them each into four fragments in the same way specified in Lemma 2.1. From this point the algorithm remains largely the same (Steps 3-4); we build the automaton for the fragments from every pattern and then proceed in the same way as algorithm CSMF. The only extra consideration is that we must be able to identify, for every fragment, the pattern from which it was extracted. To do this we alter the definition of $\mathcal{L}$ such that it now consists of tuples of the form $\left\langle p_{x_{j}^{\prime}}, \ell, j, p_{t}\right\rangle$, where $j$ identifies the pattern the fragment was extracted from; $p_{x_{j}^{\prime}}$ and $\ell$ are defined identically with respect to the pattern $x_{j}$, and $p_{t}$ remains the same. This then allows us to identify the pattern for which we must perform verification (Steps 5-6) if a fragment is matched. The verification steps are then the same as in algorithm CSMF with the respective pattern.

Theorem 4.1. Given a set $\mathcal{D}=\left\{x_{0}, x_{1}, \ldots, x_{d-1}\right\}$ of patterns of total length $M$ drawn from alphabet $\Sigma, \sigma=|\Sigma|$, and a text $t$ of length $n>\left|x_{j}\right|$, where $0 \leqslant j<d$, drawn from $\Sigma$, algorithm CDMF requires average-case time $\mathcal{O}\left(\left(1+\frac{d\left|x_{\max }\right|}{\sigma^{\frac{2 \mid x_{\min }-1}{4}}}\right) n+M\right)$ to solve the CircularDictionaryMatching problem, where $x_{\min }$ and $x_{\max }$ are the minimum- and maximum-length patterns in $\mathcal{D}$, respectively.

Proof. Constructing and partitioning the strings $x_{0}^{\prime}, x_{1}^{\prime}, \ldots, x_{d-1}^{\prime}$ from $\mathcal{D}$ can trivially be done in time $\mathcal{O}(M)$ (Steps 1-2). Building the Aho-Corasick automaton of the $4 d$ fragments requires time $\mathcal{O}(M)$; and the search time is $\mathcal{O}(n+O c c)$ (Step 3). The preprocessing step for the Ice queries on the suffixes of $T$ and $T_{r}$ can be done in time $\mathcal{O}(n)$ (Step 4). Computing $\mathcal{E}_{l}$ and $\mathcal{E}_{r}$ for each occurrence of a fragment requires time $\mathcal{O}(O c c)$ (Step 5). For each extended occurrence of some fragment $\left\langle p_{x_{j}^{\prime}}, \ell, j, p_{t}\right\rangle$, we may report $\mathcal{O}\left(\left|x_{j}\right|\right)$ valid starting positions. The expected number of occurrences for any fragment of some pattern $x_{j}$ is $n / \sigma^{\left(2\left|x_{j}\right|-1\right) / 4}$ thus $4 n / \sigma^{\left(2\left|x_{j}\right|-1\right) / 4}$ for all four fragments. So the total expected number $O c c$ of occurrences is $\sum_{j=0}^{d-1} \frac{4 n}{\sigma \mid \frac{2\left|x_{j}\right|-1}{4}}=\mathcal{O}\left(\frac{d n}{\sigma^{\frac{2\left|x_{\min }\right|-1}{4}}}\right)$, where $x_{\min }$ is the minimum-length string in $\mathcal{D}$ (Step 6). Since the expected number $O c c$ of occurrences of the fragments in $t$ is 
$\mathcal{O}\left(\frac{d n}{\sigma \frac{2\left|x_{\min }\right|-1}{4}}\right)$, algorithm CDMF requires average-case time $\mathcal{O}\left(\left(1+\frac{d\left|x_{\max }\right|}{\sigma \frac{2 x_{\min } \mid-1}{4}}\right) n+M\right)$, where $x_{\max }$ is the maximum-length string in $\mathcal{D}$.

In a similar way as in algorithm CSMF-Simple, we can apply Fact 2 to obtain algorithm CDMF-Simple and achieve the following result.

Theorem 4.2. Given a set $\mathcal{D}=\left\{x_{0}, x_{1}, \ldots, x_{d-1}\right\}$ of patterns of total length $M$ drawn from alphabet $\Sigma, \sigma=|\Sigma|$, and a text $t$ of length $n>\left|x_{j}\right|$, where $0 \leqslant j<d$, drawn from $\Sigma$, algorithm CDMF-Simple requires average-case time $\mathcal{O}\left(\left(1+\frac{d\left|x_{\max }\right|}{\sigma \frac{2\left|x_{\min }\right|-1}{4}}\right) n+M\right)$ and space $\mathcal{O}(M)$ to solve the CircularDictionaryMatching problem, where $x_{\min }$ and $x_{\max }$ are the minimum- and maximum-length patterns in $\mathcal{D}$, respectively.

Algorithm CDMF achieves average-case time $\mathcal{O}(n+M)$ iff

$$
\frac{4 d\left|x_{\max }\right|}{\sigma^{\frac{2\left|x_{\min }\right|-1}{4}}} n \leqslant c n
$$

for some fixed constant $c$. So we have

$$
\begin{gathered}
\frac{4 d\left|x_{\max }\right|}{\sigma^{\frac{2\left|x_{\min }\right|-1}{4}}} \leqslant c \\
\log _{\sigma}\left(\frac{4 d\left|x_{\max }\right|}{c}\right) \leqslant \frac{2\left|x_{\min }\right|-1}{4} \\
4\left(\log _{\sigma} 4+\log _{\sigma} d+\log _{\sigma}\left|x_{\max }\right|-\log _{\sigma} c\right) \leqslant 2\left|x_{\min }\right|-1
\end{gathered}
$$

Rearranging and setting $c$ such that $\log _{\sigma} c \geqslant 1 / 4+\log _{\sigma} 4$ gives a sufficient condition for our algorithm to achieve average-case time $\mathcal{O}(n+M)$ :

$$
\left|x_{\min }\right| \geqslant 2\left(\log _{\sigma} d+\log _{\sigma}\left|x_{\max }\right|\right)
$$

Corollary 4.1. Given a set $\mathcal{D}=\left\{x_{0}, x_{1}, \ldots, x_{d-1}\right\}$ of patterns of total length $M$ drawn from alphabet $\Sigma, \sigma=|\Sigma|$, and a text $t$ of length $n>\left|x_{j}\right|$, where $0 \leqslant j<d$, drawn from $\Sigma$, algorithm CDMF-Simple solves the CIRCULARDICTIONARYMATCHING problem in average-case time $\mathcal{O}(n+M)$ iff $\left|x_{\min }\right| \geqslant 2\left(\log _{\sigma} d+\log _{\sigma}\left|x_{\max }\right|\right)$, where $x_{\min }$ and $x_{\max }$ are the minimum- and maximum-length patterns in $\mathcal{D}$, respectively.

\section{Detailed description of CPI-II}

The index data structure CPI-II essentially consists of the suffix array SA and the inverse suffix array iSA of $t$. The result of a query for a pattern $x$ of length $m$ on the suffix array SA of $t$ of length $n$ can be represented in the form of an interval [s,e], such that the starting positions of $x$ in $t$ are given by the set $\{\mathrm{SA}[s], \mathrm{SA}[s+1], \ldots, \mathrm{SA}[e]\}$ of cardinality Occ. The query algorithm on CPI-II relies on the following two lemmas. We denote this interval by $\operatorname{lnt}_{x}^{t}$. 
Lemma 5.1 (Gusfield (1997)). Given a text $t$ of length $n$, its suffix array SA, and an interval $\mathrm{Int}_{x}^{t}$ of a query for a pattern $x$, the interval $\mathrm{Int}_{x \mathrm{c}}^{t}$ for any character $\mathrm{c}$ can be computed in time $\mathcal{O}(\log n)$.

Lemma 5.2 (Huynh et al. (2006)). Given a text $t$ of length $n$, its suffix array SA, its inverse suffix array iSA, and two intervals $\operatorname{Int}_{x_{1}}^{t}$ and $\operatorname{Int}_{x_{2}}^{t}$ of queries for patterns $x_{1}$ and $x_{2}$, respectively, the interval Int ${ }_{x_{1} x_{2}}^{t}$ can be computed in time $\mathcal{O}(\log n)$.

In order to find all the rotations of a string $x$, we can first find the intervals for all prefixes and suffixes of $x$. Let Pref[i] $=\operatorname{Int}_{x[0 . i]}^{t}$ and Suff $[i]=\operatorname{Int}_{x[i . . m-1]}^{t}$.

Consider the prefixes in ascending order by length. The interval Pref[0] for prefix $x[0]$ can be found in time $\mathcal{O}(\log n)$ by a binary search on SA. Each subsequent interval Pref $[i]$ for prefix $x[0 \ldots i]$ can be found in time $\mathcal{O}(\log n)$ by Lemma 5.1. Hence, the intervals for all prefixes can be found in time $\mathcal{O}(m \log n)$.

The intervals for the suffixes can be found in time $\mathcal{O}(m \log n)$ in a similar way. The interval Suff $[m-1]$ for suffix $x[m-1]$ can be found in time $\mathcal{O}(\log n)$. Each subsequent interval Suff $[i]$ can also be found in time $\mathcal{O}(\log n)$ by first finding $\operatorname{Int}_{\mathrm{c}}^{t}$, where $\mathrm{c}=x[i]$, in

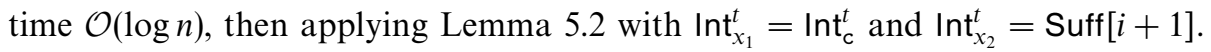

Having found the intervals Pref $[i]$ and Suff $[i]$ for all $i \in[0, \ldots, m-1]$, we next find the set of intervals for all the rotations of $x$. The interval for the first rotation of $x$ is Suff $[m-1]$. For $j \in[0, \ldots, m-1]$, the interval for the $j$-th rotation of $x$ can be found in time $\mathcal{O}(\log n)$ using Lemma 5.2 with $\operatorname{Int}_{x_{1}}^{t}=\operatorname{Suff}[j]$ and $\operatorname{Int}_{x_{2}}^{t}=\operatorname{Pref}[(j+m-2) \bmod (m-1)]$. Thus all intervals for all the rotations of $x$ can be found in time $\mathcal{O}(m \log n)$. Finally, we use SA to convert this set of intervals into a set of occurrences in $t$ of cardinality $O c c$. In all, this query algorithm requires time $\mathcal{O}(n+m \log n+O c c)$.

\section{Experimental results}

We implemented algorithms CDMF-Simple and the index data structure CPI-II as library functions to perform circular string matching for a set of patterns. The functions were implemented in the $\mathrm{C}$ programming language and developed under GNU/Linux operating system. They take as input arguments a set $\mathcal{D}=\left\{x_{0}, x_{1}, \ldots, x_{d-1}\right\}$ of patterns and a text $t$; and then return the list of starting positions of the occurrences of the rotations of $x_{0}, x_{1}, \ldots, x_{d-1}$ in $t$ as output. The library implementation of CDMF-Simple is distributed under the GNU General Public License (GPL), and it is available at http://www.inf.kcl.ac.uk/research/projects/asmf/, which is set up for maintaining the source code and the man-page documentation. The library implementation of CPI-II is distributed under the GPL, and it is available at http://github.com/blandw/cpm. The experiments were conducted on a Desktop PC using one core of Intel i7 $2600 \mathrm{CPU}$ at $3.4 \mathrm{GHz}$ under GNU/Linux. The programmes were compiled with gcc version 4.6 .3 at optimization level 3 (-O3). Time and memory measurements were taken using the GNU/Linux time command.

To evaluate the efficiency of CDMF-Simple, we compared its performance against CPI-II using real data. As input datasets we used: for the set of patterns, a set of $d=22,918$ Sugarcane white streak virus short reads, produced by Illumina platform, of total length 
Table 1. Elapsed-time and speed-up comparisons of algorithms CPI-II and CDMF-Simple using synthetic DNA data $(\sigma=4)$ for $n=100 \mathrm{MB}$.

\begin{tabular}{ccccc}
\hline $\begin{array}{c}\text { Number of } \\
\text { patterns }\end{array}$ & $\begin{array}{c}\text { Length of } \\
\text { each pattern }\end{array}$ & CPI-II (s) & CDMF-Simple (s) & $\begin{array}{c}\text { Speed-up of } \\
\text { CDMF-Simple }\end{array}$ \\
\hline 10 & 25 & 8.5 & 4.9 & 1.73 \\
100 & 25 & 8.6 & 5.1 & 1.68 \\
1000 & 25 & 8.6 & 5.6 & 1.53 \\
10,000 & 25 & 9.0 & 6.2 & 1.45 \\
10 & 50 & 8.4 & 4.3 & 1.95 \\
100 & 50 & 8.6 & 5.1 & 1.68 \\
1000 & 50 & 8.6 & 5.7 & 1.50 \\
10,000 & 50 & 9.4 & 6.2 & 1.51 \\
10 & 100 & 8.6 & 4.3 & 2 \\
100 & 100 & 8.6 & 4.9 & 1.75 \\
1000 & 100 & 8.7 & 5.8 & 1.50 \\
10,000 & 100 & 9.6 & 7.2 & 1.33 \\
\hline
\end{tabular}

$M=516,076$ base pairs; and, for the text, the Homo sapiens chromosome 1 sequence of length $n=248,956,422$ base pairs. CPI-II finished the assignment in 25.1 seconds; CDMF-Simple finished in 17.7 seconds. The maximum allocated memory was $2436 \mathrm{MB}$ for CPI-II and $691 \mathrm{MB}$ for CDMF-Simple. As input datasets we also used: for the set of patterns, a set of $d=14,880$ Human immunodeficiency virus (HIV-1) short reads, produced by 454 Life Sciences platform, of total length $M=3,040,354$ base pairs; and, for the text, the Homo sapiens chromosome 1 sequence of length $n=248,956,422$ base pairs. CPI-II finished the assignment in 30.1 seconds; CDMF-Simple finished in 25.5 seconds. The maximum allocated memory was $2436 \mathrm{MB}$ for CPI-II and $675 \mathrm{MB}$ for CDMF-Simple.

To further evaluate the efficiency of CDMF-Simple, we compared its performance against CPI-II using synthetic data. The data were generated using a randomized script; and the parameters for this script were chosen based on the properties (length) of real data. Tables 1 and 2 illustrate elapsed-time and speed-up comparisons for different combinations of input parameters. As it is demonstrated by the experimental results, algorithm CDMF-Simple is in all cases the fastest with a speed-up improvement between 1.3 and 2.3 over CPI-II. The maximum allocated memory (per task) for the experiments in Table 1 was $998 \mathrm{MB}$ for CPI-II and 337 MB for CDMF-Simple. The maximum allocated memory (per task) for the experiments in Table 2 was $1940 \mathrm{MB}$ for CPI-II and $525 \mathrm{MB}$ for CDMF-Simple. Notice that, this occurs for the largest number of patterns, and CDMFSimple has a lower memory footprint for less patterns, whilst CPI-II remains static. This confirms our theoretical findings in terms of space complexity.

Here, it becomes evident what is generally expected in practical terms: there is a certain point, as the value of $M$ grows and $n$ remains static, that CDMF-Simple will become slower than CPI-II; however, for smaller $M$, CDMF-Simple is expected to retain a significant speed-up, in particular when one has multiple online query texts. 
Table 2. Elapsed-time and speed-up comparisons of algorithms CPI-II and CDMF-Simple using synthetic DNA data ( $\sigma=4)$ for $n=200 \mathrm{MB}$.

\begin{tabular}{ccccc}
\hline \hline $\begin{array}{c}\text { Number of } \\
\text { patterns }\end{array}$ & $\begin{array}{c}\text { Length of } \\
\text { each pattern }\end{array}$ & CPI-II (s) & CDMF-Simple (s) & $\begin{array}{c}\text { Speed-up of } \\
\text { CDMF-Simple }\end{array}$ \\
\hline 10 & 25 & 21.1 & 10.4 & 2.02 \\
100 & 25 & 21.3 & 10.8 & 1.97 \\
1000 & 25 & 21.4 & 11.9 & 1.79 \\
10,000 & 25 & 21.5 & 13.3 & 1.61 \\
10 & 50 & 21.2 & 9.2 & 2.3 \\
100 & 50 & 21.5 & 10.8 & 1.99 \\
1000 & 50 & 21.5 & 12.3 & 1.74 \\
10,000 & 50 & 22.3 & 13.4 & 1.66 \\
10 & 100 & 21.3 & 9.1 & 2.34 \\
100 & 100 & 21.6 & 10.4 & 2.07 \\
1000 & 100 & 22 & 12.4 & 1.77 \\
10,000 & 100 & 22.3 & 15.3 & 1.45 \\
\hline
\end{tabular}

\section{Final remarks}

In this article, we presented a suboptimal average-case algorithm for circular string matching requiring time $\mathcal{O}(n)$ and space $\mathcal{O}(m)$. We showed how it can be extended to solve the circular dictionary-matching problem in average-case time $\mathcal{O}(n+M)$ and space $\mathcal{O}(M)$, assuming that the shortest pattern is sufficiently long. Experimental results, using real and synthetic data, demonstrate that the implementation of the presented algorithms can accelerate the computations by more than a factor of two compared to the corresponding implementation of other approaches. For future work, we will try to improve our algorithms in order to achieve average-case optimality; and to extend our approaches to the edit distance model (Barton et al. 2014, 2015).

The publication costs for this article were funded by the Open Access funding scheme of King's College London. Tanver Athar is supported by an EPSRC grant (Doctoral Training Grant \#EP/L504798/1). Solon P. Pissis is supported by a Research Grant (\#RG130720) awarded by the Royal Society.

\section{References}

Aho, A. V. and Corasick, M. J. (1975). Efficient string matching: An aid to bibliographic search. Communications of the ACM 18(6) 333-340.

Barton, C., Iliopoulos, C. S. and Pissis, S. P. (2013). Circular string matching revisited. In: Proceedings of the 4th Italian Conference on Theoretical Computer Science (ICTCS 2013) 200-205. 
Barton, C., Iliopoulos, C. S. and Pissis, S. P. (2014). Fast algorithms for approximate circular string matching. Algorithms for Molecular Biology 9 (9). Available at http://www. almob.org/content/9/1/9.

Barton, C., Iliopoulos, C. S. and Pissis, S. P. (2015). Average-case optimal approximate circular string matching. In: Dediu, A.-H., Formenti, E., Martin-Vide, C. and Truthe, B. (eds.) Language and Automata Theory and Applications, Lecture Notes in Computer Science, volume 8977 Springer, Berlin 85-96.

Belazzougui D. (2010). Succinct dictionary matching with no slowdown. In: Amir, A. and Parida, L. (eds.) Combinatorial Pattern Matching, Lecture Notes in Computer Science, volume 6129 Springer, Berlin 88-100.

Chan, H., Hon, W., Lam, T. and Sadakane, K. (2007). Compressed indexes for dynamic text collections. ACM Transactions on Algorithms 3 (2). Available at http://dl.acm.org/ citation. cfm?doid=1240233.1240244.

Chen, K., Huang, G. and Lee, R. C. (2013). Bit-parallel algorithms for exact circular string matching. Computer Journal 57 (5) 731-743.

Dori, S. and Landau, G. M. (2006). Construction of Aho Corasick automaton in linear time for integer alphabets. Information Processing Letters 98 (2) 66-72.

Fischer, J. (2011). Inducing the LCP-array. In: Dehne, F., Iacono, J. and Sack, J.-R. (eds.) Algorithms and Data Structures, Lecture Notes in Computer Science, volume 6844, Springer, Berlin 374-385.

Fischer, J. and Heun, V. (2011). Space-efficient preprocessing schemes for range minimum queries on static arrays. SIAM Journal on Computing 40 (2) 465-492.

Fredriksson, K. and Grabowski, S. (2009). Average-optimal string matching. Journal of Discrete Algorithms 7 (4) 579-594.

Frousios, K., Iliopoulos, C. S., Mouchard, L., Pissis, S. P. and Tischler, G. (2010). REAL: An efficient REad ALigner for next generation sequencing reads. In: Proceedings of the First ACM International Conference on Bioinformatics and Computational Biology, BCB 10, USA, ACM 154159.

Gusfield D. (1997). Algorithms on Strings, Trees and Sequences, Cambridge University Press.

Hon, W., Ku, T., Shah, R. and Thankachan, S. V. (2013). Space-efficient construction algorithm for the circular suffix tree. In Fischer, J. and Sanders, P. (eds.) Combinatorial Pattern Matching, Lecture Notes in Computer Science, volume 7922, Springer, Berlin 142-152.

Hon, W., Ku, T., Shah, R., Thankachan, S. V. and Vitter, J. S. (2010). Faster compressed dictionary matching. In: Chavez, E. and Lonardi, S. (eds.) String Processing and Information Retrieval, Lecture Notes in Computer Science, volume 6393, Springer, Berlin 191-200.

Hon, W., Lu, C., Shah, R. and Thankachan, S. V. (2011). Succinct indexes for circular patterns. In Asano, T., Nakano, S.-I., Okamoto, Y. and Watanabe, O (eds.) Algorithms and Computation, Lecture Notes in Computer Science, volume 7074, Springer, Berlin 673-682.

Huynh, T. N. D., Hon, W., Lam, T. and Sung, W. (2006). Approximate string matching using compressed suffix arrays. Theoretical Computer Science 352 (1) 240-249.

Ilie, L., Navarro, G. and Tinta, L. (2010). The longest common extension problem revisited and applications to approximate string searching. Journal of Discrete Algorithms 8 (4) 418-428.

Iliopoulos, C. S. and Rahman, M. S. (2008). Indexing circular patterns. In: Nakano, S.-I. and Rahman, Md. S. (eds.) WALCOM: Algorithms and Computation, Lecture Notes in Computer Science, volume 4921, Springer, Berlin 46-57.

Lothaire, M. (ed.) (2005). Applied Combinatorics on Words, Cambridge University Press.

Manber, U. and Myers, E. W. (1993). Suffix arrays: A new method for on-line string searches. SIAM Journal on Computing 22 (5) 935-948. 
Nong, G., Zhang, S. and Chan, W. H. (2009). Linear suffix array construction by almost pure induced-sorting. In: Storer, J. A. and Marcellin, M. W. (eds.) Proceedings of the 2009 Data Compression Conference, DCC 09, Washington, DC, USA, IEEE Computer Society 193-202.

Rivest, R. (1976). Partial-match retrieval algorithms. SIAM Journal on Computing 5 (1) 19-50.

Smyth, B. (2003). Computing Patterns in Strings. Pearson, Addison-Wesley.

Weiner, P. (1973). Linear pattern matching algorithms. In: Proceedings of the 14th Annual Symposium on Switching and Automata Theory (SWAT 1973), IEEE Computer Society 1-11.

Wu, S. and Manber, U. (1992). Fast text searching: Allowing errors. Communications of the ACM 35 (10) 83-91. 